litische Meinungen offen zu vertreten (S. 4). Nach einer Untersuchung der äußeren Rahmenbedingungen (Hochschulwesen, politischer Prozeß, politische Kultur), Kap. I, die auch die Prinzipien des Systems der Neuen Ordnung in Indonesien behandelt (29-34), gibt der Verfasser einen Uberblick über die bisherigen Studentenbewegungen in der jungen indonesischen Geschichte (Kap. II). Hauptteil der vorliegenden Untersuchung ist jedoch eine Analyse der politischen Einstellungen unter den Studenten sowie des Einflusses der sozio-demographischen Merkmale auf diese Einstellungen (Kap. III). Zielgruppe der in den Jahren 1971 und 1972 durchgeführten Befragung waren die Studenten der Hochschulen Jakarta, Jogjakarta und Bandung. Das mittels eines Fragebogens (S. 179) erhobene Datenmaterial erstreckt sich nicht auf die Variable ,,politisches Verhalten der Studenten“, da der Verfasser angesichts der derzeitigen politischen Situation in Indonesien die Untersuchung dann gefährdet sah (S. 44). Im Anschluß an eine Zusammenfassung der Befragungsergebnisse (S. 131) erstellt der Verfasser mit Hilfe der Cluster-Analyse Einstellungsprofile in der Studentenschaft (S. 134). Die Messung der Befragungsergebnisse an den eingangs postulierten Einstellungsmerkmalen führt den Verfasser zu dem Schluß, daß die indonesische Studentenschaft den Anspruch des politischen Korrektivs zur Zeit nicht erfüllt (S. 159).

Ulrich Rausch

\title{
ILO
}

\section{Poverty and Landlessness in Rural Asia}

Geneva 1977, 288 S.

In dieser Edition wird hervorragendes Material zur These der strukturellen Heterogenität zusammengetragen, d. h. zu dem Postulat, daß trotz (z. T. beachtlichen) Wachstums der Agrarproduktion nicht nur die Ungleichheit in der ländlichen Gesellschaft verschärft wird, sondern die Armutsgruppen meist auch absolut ärmer geworden sind. Der Band versammelt Fallstudien über Pakistan (S. M. Naseem), Bangladesh und die Philippinen (A. R. Khan), Sri Lanka und West Malaysia (E. H. L. Lee), Java (I. Palmer) und über die indischen Bundesstaaten Punjab (J. Rajaraman), Uttar Pradesh und Bihar (R. Nayyar) sowie Tamil Nadu (C. T. Kurien). Einleitend werden in einem zusammenfassenden Essay auch die Ursachen für die Verschärfung der Situation für die Landarmut untersucht. Der ungleichmäßige Zugang zu den Produktionsmitteln, insbesondere dem Land, und, damit zusammenhängend, der unzureichenden Ausnutzung vorhandener Ressourcen (Arbeitskraft, Land, Kapital) sowie der Bias zu kapitalintensiven Investitionen, werden dabei an erster Stelle herausgearbeitet. Der Band wird durch einen (alternativen) Beitrag über die Stratifizierung der ländlichen Einkommen in der VR China abgeschlossen (von A. R. Khan). In ihm wird - auf einer allerdings wesentlich schlechteren Datenbasis als in den anderen Länderbeiträgen - deutlich gemacht, in welcher Weise durch die Landreform hier die ländlichen Einkommen egalisiert worden sind, welche beträchtlichen, wenn auch im Vergleich zum vorrevolutionären China und den anderen untersuchten Ländern geringen, Einkommensunterschiede auch danach noch bestanden und durch welche (z. T. erfolglosen) Maßnahmen versucht wurde, diese weiter einzuebnen. Für den Nicht-Marxisten mögen Einkommensunterschiede an sich noch nicht bedenklich sein, würden sie nicht, wie in den hier untérsuchten peripher-kapitalistischen Ländern, mit einer absoluten Armut verbunden sein, die großen Bevölkerungsgruppen den Konsum der Grundbedürfnisse verwehren würde (d. h. bei allgemein relativ hohen Einkommen aller Bevölkerungsgruppen könnten auch erhebliche Einkommensunterschiede tragbar sein). Trotz allgemein offenbar geringer Einkommenshöhe in der VR China, scheint dort die Deckung der menschlichen Grundbedürfnisse - nach Khan - gewährleistet zu sein. 
Inwieweit das chinesische System darüber hinaus allerdings aúch noch eine allmähliche Einkommens- und Konsumsteigerung erlaubt, wird hier leider ausgeklammert.

Bedauerlich auch, daß die offenbar relativ erfolgreichen Modelle peripher-kapitalistischer Entwicklung in Asien, Taiwan und Süd-Korea, in diesem Band nicht berücksichtigt und den anderen Fallstudien gegenübergestellt wurden. Dennoch, es handelt sich bei dieser ILOStudie um einen für die Entwicklungstheorie und Entwicklungspolitik sehr lesenswerten Beitrag.

Rolf Hanisch

\section{ERIKA KNABE}

\section{Frauenemanzipation in Afghanistan.}

Ein empirischer Beitrag zur Untersuchung von sozio-kulturellem Wandel und sozio-kultureller Beständigkeit.

Afghanisçhe Studien, Bd.16, Meisenheim am Glan: Verlag Anton Hein 1977, 471 S., 74,-DM.

In einem Zeitraum von mehr als sechs Jahren sammelte die Autorin, die eine der offiziellen Landessprachen - Dari - beherrscht, die Daten zu der vorliegenden Studie - vorwiegend in Kabul. Eine ausführliche Auseinandersetzung mit der islamischen Glaubenslehre - mit vielen Textbelegen - soll die weitgehend durch den Islam festgeschriebene Stellung der Frau in den einzelnen Rollen darlegen. Nach Aussage der Verfasserin hat sich nur ein Bruchteil der afghanischen Frauen (0,4 v.H., s.S. 143) von diesen bis heute gültigen Lebensformen bis zu einem gewissen Grad freimachen können. Die gescheiterten Reformen des westlich orientierten Königs Amanullah (1919 - 1929) haben die Liberalisierung der Stellung der Frau um fast 50 Jahre verzögert. An Hand von Straßenzählungen in einzelnen Stadtteilen Kabuls und Herats und an Hand von Befragungen, sowie durch Auswertung von autobiographischen Notizen von Hörerzuschriften an Radio Kabul versucht die Autorin den sozio-kulturellen Wandel bzw. die Beständigkeit der bestehenden Normen zwischen den Geschlechtern in der Gesellschaft nachzuweisen. Durch die Studie wird nachgewiesen, was für die meisten Entwicklungsländer zutrifft, daß Frauen der modernen Kabuler Familien, also hauptstädtische Ober- und Mittelschicht, sich Freiräume innerhalb und außerhalb der Familie geschaffen haben, daß sie aber kaum als Entwicklungsagenten fungieren bzw. akzeptiert werden. Es wird nicht erwähnt, daß diese Gruppe von Frauen oft auch nicht daran interessiert sind, Innovationsträger zu sein, da sie damit soziale Strukturen ins Wanken bringen und ihre bestehenden Privilegien verlieren könnten. Die Studie ist durch die verschiedenen Erhebungsmethoden ausgesprochen reizvoll, doch bleibt zu fragen, warum in einem so langen Untersuchungszeitraum nicht die ,Masse' der Kabuler Frauen, ganz abgesehen von der Landbevölkerung, mehr in die Arbeit einbezogen werden konnte. Der Titel der Studie weckt beim Leser diese Erwartung.

Gudrun Martius-von Harder 